\title{
Conformational Changes in Solution Multimers of Dynamin-related Protein 1 (Drp1) Facilitate Functional Assembly
}

Kristy Rochon ${ }^{1}$, Anibal Tornes Blanco ${ }^{2}$ and Jason Mears ${ }^{3}$

${ }^{1}$ The Cleveland Center for Membrane and Structural Biology, School of Medicine, Case Western Reserve University, Cleveland, Ohio, United States, ${ }^{2}$ School of Medicine, Case Western Reserve University, Cleveland, Ohio, United States, ${ }^{3}$ Case Western Reserve University, Cleveland, Ohio, United States

Mitochondrial dynamics are essential for cellular health, and an imbalance in this cycle has been implicated in many diseases, from heart failure [1] to prion-related neurodegeneration [2]. Several proteins are critical for mitochondrial dynamics, and paradoxically, both hyper- and hypofission can lead to human pathologies [3,4]. Dynamin-related protein 1 (Drp1) is the master regulator of mitochondrial fission [5,6]; however, the underlying mechanisms governing this regulation are largely unclear.

Drp1 is comprised of three conserved domains common to the dynamin superfamily: the GTPase domain or G-domain, the GTPase effector domain (GED), and the middle domain. The GED and middle domain together form the self-assembly region referred to as the stalk [7]. In solution, Drp1 forms distinct multimers in a concentration dependent manner. There is evidence that larger oligomers must cycle down to a dimer state to assemble into helical structures around the mitochondria outer membrane and mediate constriction [8]. We have observed that Drp1's hinge 1, a disordered region connecting the GTPase domain and the stalk, confers a high degree of flexibility in dimer populations. In larger oligomers, this flexibility is reduced, leading to more homogenous conformations. We hypothesize that these stabilized oligomers are a storage form of the cytosolic protein, while the flexibility observed in the dimer facilitates the conformational rearrangement needed for helical assembly (Fig. 1).

Point mutations in Drp1 have been shown to enrich higher-order oligomers, and these changes are associated with defective mitochondrial fission [9,10]. Conversely, separate point mutations cannot selfassemble, and Drp1 forms an obligate dimer [11]. We have used these mutants and wild-type protein to identify structural differences in solution multimers of Drp1. By resolving high resolution cryoEM structures of the Drp1 dimer, tetramer, and hexamer, as well as helical polymers on lipid templates, we will elucidate conformational changes that facilitate functional assembly. In addition to electron microscopy, we are using size exclusion chromatography with multiple angle light scattering (SECMALS) and molecular dynamic simulations (MDS) to identify critical residues and interfaces that regulate oligomerization and conformation flexibility to control assembly of the mitochondrial fission machinery.

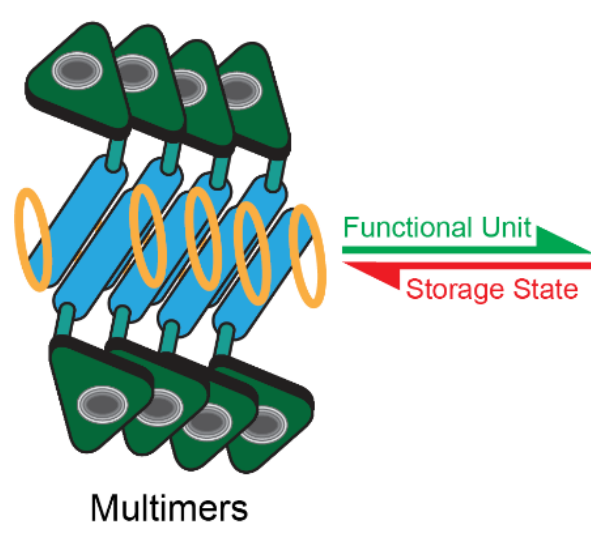

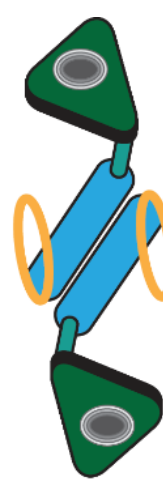

Dimer

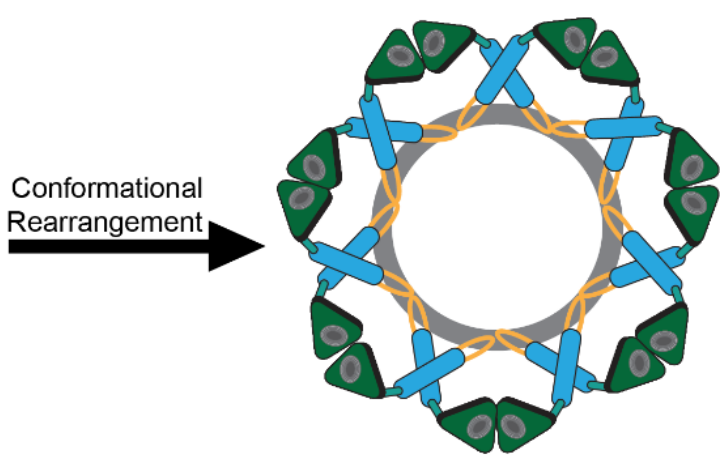

Helical Assembly 
Figure 1. In the cytosol, Drp1 multimers serve as a storage form. These multimers cycle down to the functional unit, an assembly-competent dimer. Dimers then form helical polymers on the mitochondria outer membrane to mediate constriction.

\section{References}

1. S. M. Adaniya, J. O-Uchi, M. W. Cypress, Y. Kusakari and B. S. Jhun. Am J Physiol Cell Physiol, 316(5), C583-C604 (2019) doi:10.1152/ajpcell.00523.2018

2. T. Zhu, J. L. Chen, Q. Wang, W. Shao and B. Qi. Front Aging Neurosci, 10, 336 (2018) doi:10.3389/fnagi.2018.00336

3. J. A. W. Heymann and J. E. Hinshaw. Journal of cell science, 122(Pt 19), 3427-3431 (2009) doi:10.1242/jcs.051714

4. S. Gao, A. von der Malsburg, S. Paeschke, J. Behlke, O. Haller, G. Kochs and O. Daumke. Nature, 465(7297), 502-506 (2010) doi:10.1038/nature08972 [doi]

5. E. Smirnova, D. L. Shurland, S. N. Ryazantsev and A. M. van der Bliek. The Journal of cell biology, 143(2), 351-358 (1998)

6. E. Smirnova, L. Griparic, D. L. Shurland and A. M. van der Bliek Molecular biology of the cell, 12(8), 2245-2256 (2001)

7. C. Fröhlich, S. Grabiger, D. Schwefel, K. Faelber, E. Rosenbaum, J. Mears, O. Rocks and O. Daumke. The EMBO Journal, 32(9), 1280-1292 (2013) doi:10.1038/emboj.2013.74

8. B. Lu, B. Kennedy, R. W. Clinton, E. J. Wang, D. McHugh, N. Stepanyants, P. J. Macdonald, J. A. Mears, X. Qi and R. Ramachandran. Sci Rep, 8(1), 10879 (2018) doi:10.1038/s41598-018-29001-9

9. P. J. Macdonald, N. Stepanyants, N. Mehrotra, J. A. Mears, X. Qi, H. Sesaki and R. Ramachandran. Molecular biology of the cell, 25(12), 1905-1915 (2014) doi:10.1091/mbc.E14-02-0728 [doi]

10. R. W. Clinton, C. A. Francy, R. Ramachandran, X. Qi and J. A. Mears. The Journal of biological chemistry, 291(1), 478-492 (2016) doi:10.1074/jbc.M115.680025 [doi]

11. C. R. Chang, C. M. Manlandro, D. Arnoult, J. Stadler, A. E. Posey, R. B. Hill and C. Blackstone. The Journal of biological chemistry, 285(42), 32494-32503 (2010) 